\title{
DASHCAM AS A DEVICE TO INCREASE THE ROAD SAFETY LEVEL Veronika Adamová ${ }^{1}$
}

\begin{abstract}
In recent years, there has been a huge boom in the market in terms of both supply and demand for dashcams. This stems from the purpose of the camera installed in the vehicle. Dashcams are currently becoming an important aid in solving various adverse incidents, such as detecting violations of traffic regulations and other laws and recording and clarifying the course of a traffic accident or other criminologically relevant event. The purpose of this camera is to monitor the space in front and behind the vehicle, or the situation inside the vehicle. The content of this article is aimed at acquainting the reader with basic information about dashcams. Specifically, the individual parts of the article will discuss the importance and significance of the dashcams, the benefits of its use, and the legislative framework that regulates its use. The paper will conclude with a brief overview of innovative approaches based on the application of a dashcam for the purpose of increasing the level of safety of road users while driving a vehicle.
\end{abstract}

UDC Classification: 629.06, DOI: https://doi.org/10.12955/pns.v1.113

Keywords: criminologically relevant event, dashcam, digital evidence, safety, traffic accident

\section{Introduction}

The target group of cameras discussed in this paper are dashcams (referred to as on-board cameras, dashboard cameras or vehicle cameras in foreign literature). The enormous boom and frequent implementation of dashcams are presented in several papers describing the benefits of their use (Ma, Reibman, 2016; Mehrish et al., 2017; Kadu et al., 2018). Dashcams form a specific subset of video surveillance systems, recording situations on the road through the vehicle's windshield. The main reason for their use being to ensure vehicle safety. In particular, vehicle safety means recording traffic accidents or other events related to road safety in an objective way and thus reducing the uncertainty in witness testimonies and misleading experts. Another advantage of dashcams recordings is that they provide support to insurance companies to solve questions of damage compensation more efficiently. Motivations to install a dashcam can be various e.g. advantageous insurance premium to drivers offered by the insurance companies, gathering evidence, that can become evidence in judicial proceedings (Śtitilis, Laurinaitis, 2016). Torney (2014) write, that video recording from an installed camera in a vehicle helps insurance companies to deal more quickly with damages issues, in many countries of the EU insurance companies offer their policyholders discounts on premiums.

Despite the existence of potential threats, e.g. personal privacy threats and interference with citizens' personal data, video recordings from dashcams help to achieve the right goals and defend the citizen (Jones, 2014; Gusner, 2014). In addition, numerous instances of introducing cameras into user's vehicles have made automotive developers implement cameras to vehicle as one of the basic element of vehicle equipment. This means not only recording the image while driving, but also evaluating it automatically to minimize risk to the occupants of the vehicle. The example could be Russo's research (Russo et al., 2015) where there are described two different algorithms (one for identifying the state of the semaphore and the other for tracking and recognizing vehicles) in near real time, using the data recorded by the dashcam.

The digital footprint, such as the video recording from the dashcam is the most valuable resource about the course of an accident or other criminologically relevant events (CREs), because it is possible to extract not only quantitative data on the movement of road vehicles, but also information on the spatiotemporal context or information about the driver's vision conditions (Kolla et al., 2019). It is the source of objective information for possible later purposes of expert image analyzing and forensic casework (Wong at al., 2014; Han, 2016).

The aim of this paper is to familiarize the reader with the issue of dashcams based on general knowledge. Its individual parts will discuss the basic history of dashcams and the legislative framework within the European Union (EU) that regulates their use. The importance and benefits of their use will be demonstrated on the results from research by Aviva (Aviva, 2018).

\footnotetext{
${ }^{1}$ University of Žilina, Faculty of Security Engineering, Department of Security Management, Slovakia, veronika.adamova@fbi.uniza.sk
} 


\section{A brief overview of the dashcam development}

The earliest use of dashcams is attributed to the US state of Texas in the 1980s (Pettie, 2013). The reason for their use was a video recording of the activities of police officers working in remote areas, which should increase their security. However, the first installations of these cameras in police vehicles were expensive and inefficient. In the 1990s, a second wave came and dashcams began to be installed in police vehicles, but in this case for the purpose of monitoring and supervising compliance with the rights and obligations of police officers and preventing abuse of their status. The videos then served as evidence in the case of a police officer accused of improper practice (Štitilis, Laurinaitis, 2016).

The third wave is mainly associated with Russia. The cheaper and more affordable technology has started their mass purchase by ordinary vehicle users. This was due to frequent insurance frauds that drivers wanted to protect themselves from in order to avoid false accusations of causing an accident and to minimize the risk of being involved in traffic accidents (Lavrinc, 2013).

Today, dashcams are becoming increasingly popular (Štitilis, Laurinaitis, 2016; Mehrish et al., 2017). The benefit of camera recording is to store relevant information on the course of the negative event in real time and space on the camera's storage medium. A negative event in terms of traffic accidents, parking accidents, insurance fraud, road crime or other CREs almost always has a destructive effect on the health or life of persons, property or the environment (Šoltés et al., 2019).

\section{Legal regulations of the use of dashcams in the $\mathrm{EU}$ conditions}

In connection with the development of video surveillance systems (Boroš et al., 2018) it is possible to talk about their significant changes not only in the area of technical and technological progress, but also in the sphere of legislative regulation. The use of cameras and the production of video recordings is subject to a number of legal standards. In this respect, it is worth to highlighting Directive 95/46/EC of the European Parliament and of the Council and Regulation 2016/679 of the European Parliament and of the Council (GDPR), which regulate privacy and personal data protection measures.

Aspects of privacy in connection with the use of dashcams were discussed in the article (Štitilis, Maurinaitis, 2016). The study focused on analyzing and comparing the current state of the legislation on the use of dashcams in selected EU countries (Austria, Germany, Luxembourg, United Kingdom, Belgium and Lithuania). On the basis of an empirical analysis of relevant legal documents, case law, decisions and opinions responsible for data protection in selected countries, there is no unanimous view regarding the legality of the use of dashcams, despite the unified EU personal data protection system. According to the authors, the use of such cameras as well as the publication of records should not be prohibited. However, their application must strictly observe the principles of expediency, proportionality and privacy, including related legal standards. The authors attribute the necessity to create a clear wording of the legislation on the use of cameras in vehicles, in particular for reasons of prevention and protection of private and public interest. This includes investigating traffic accidents, fraud protection, parking accidents, investigating traffic offenses, recording drivers' activities, capturing unexpected events, illegally paying of fines and others. Possible support for solving the problem of different vehicle camera regulation is seen by the authors in cooperation with the manufacturers of these cameras, who can technically and using programs limit the potential misuse of video recording.

The operation of dashcams and the use of their records in criminal and minor offense proceedings is mentioned in the paper by Ivor and Kolla (2019). The paper points out the probative power of video recording from a camera in a vehicle, which permanently monitors the road traffic in front of the vehicle while driving, in some cases the situation in the vehicle and behind the vehicle. Thus, in the event of a collision, the relevant video record becomes objective and indisputable evidence. The article further analyzes the legislative framework of the Slovak Republic within the use of vehicle cameras and video recordings. The term dashcam does not appear in any legislation. The importance of such records is not only attributed to the visual capture of the CRE but also to the causal, temporal and spatial context of the investigated CRE (e.g. the situation that preceded the accident, the mechanism of the accident, the causes and consequences of the accident) and thereby providing evidence for technical analysis and reconstruction of accident events by road traffic experts.

In accordance with the GDPR, it can be declared that when using recordings from dashcams it is necessary to ensure that the privacy of persons who are not unduly interfered with, the interventions must only be proportionate to the purpose of their use. A party who is guilty or injured is in law if it 
provides evidence in the context of an accident clarification, and this is evidenced by video surveillance. On the basis of the evidence it is then possible to identify the fault of the accident and to punish the right offender. It is natural that if a video recording records the facts of the incident being investigated, the disposing person will hand over such material to the competent parties (Ivor, Kolla, 2019).

\section{Methodology}

The importance and significance of the use of dashcams are based on the results of the research from Aviva in June 2018 (Aviva, 2018). This research was carried out in the form of survey and applied to UK motorists. 2438 respondents of UK motorists represent representative sample. Due to the fact that the results are taken over of other research, it is not possible to provide detailed information on its processing. The outputs of this survey serve as supporting data for the demonstration and showing of the significance of the dashcam as a device that can increase the level of road safety. The obtained data was processed by using tools in MS Excel. The outcomes are presented in the next part of the paper, where graphically processed statistical data and its interpretation can be found. All numerical data are taken from the survey mentioned above (Aviva, 2018).

\section{Results and discussion}

From the representative sample, almost 660 (27\%) of them currently use dashcams in their vehicles. Nearly half of them (43\%) began to use this device in the past 1 year. It shows, that at the turn of years 2017 and 2018, there was an increase in popularity and interest in dashcams. The opinions and statements of dashcam users are presented in the following Figure 1 which shows the reasons that led motorists to buy and implement dashcams in their vehicles. The respondents agree with more than one reason for their decision to buy a dashcam. Approximately 112 respondents (17\%) said that the reason for purchasing this device was a previous negative experience on the road. It was mainly about the events, when the traffic accident participants were trying to prove, that they were not a fault in the traffic accident. Almost half of dashcam users (48\%) use dashcam because of peace of mind, because if necessary, they are able to share the footage and explain what happened. More than a third (36\%) said, that they feel safer on the road during driving. According to the results, the drivers mention the use of the dashcam as one of the key safety factors on the road during their driving.

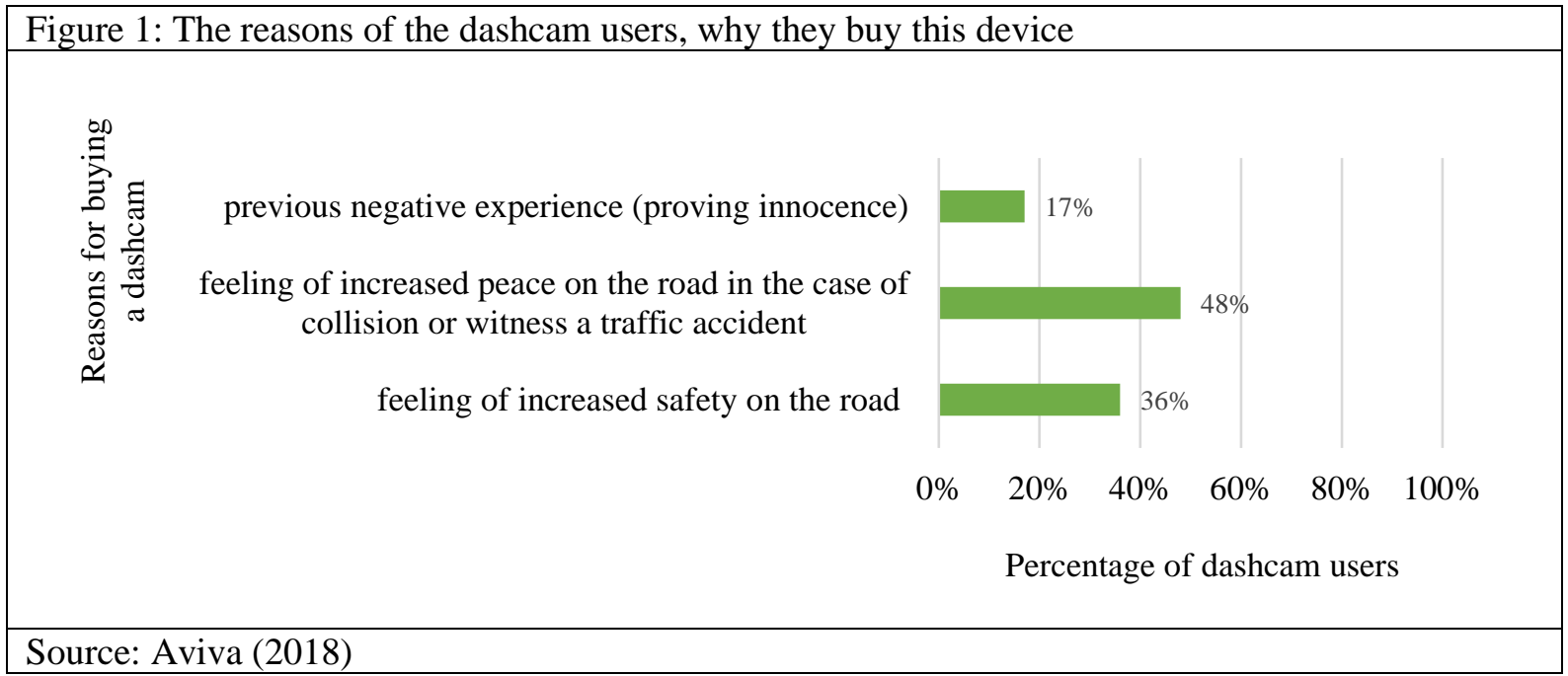

In the Figure 1, several reasons are shown why a camera in a vehicle is useful and important. In order for the dashcam video recording to be used in criminal or offense proceedings, it must meet certain requirements. There is a need, not only in terms of traffic accident clarification, but also general safety, for the camera to meet all the necessary parameters and to be able to record and save video. In general, dashcams should meet the following requirements - high-quality resolution, focused and detailed optical recording, quality recording in low light conditions, sufficient frame rate, recording at immediate impact (during and after the impact), the ability to save and store the recording after impact and the ability to record and preserve data in case of severe mechanical damage caused to the camera. The survey (Aviva, 2018) revealed that almost two-thirds of dashcam users (62\%) have experience with using footage to improve their insurance claims process and solve traffic accidents or other CREs. For around 131 users (32\%), the digital track captured by the dashcam was helpful in proving their innocence. It should be 
noted, that recorded traffic accidents or other CRE can help other event participant, e.g. when the dashcam user is in the position of a witness of the event, and the incriminated event is captured on his dashcam. The research results from Aviva revealed more than 140 cases (22\% of dashcam users), when dashcam users were witnesses in a traffic accident and the event was recorded by their dashcam. These results are graphically presented in Figure 2.

\begin{tabular}{|l} 
Figure 2: Percentage redistribution of dashcam users and use footage in claims processes \\
drivers without \\
dashcam (73\%)
\end{tabular}

Based on research from Aviva, a growing number of drivers are seeking help in vehicle cameras to keep them safe during driving. There are several logical reasons for road vehicle drivers to buy and install dashcams. The digital footprint as a result of the recording can be used in a wide spectrum, ranging from dealing with minor events (minor traffic and parking accidents, invalid and illegal fines, insured events...) to complicated forensic investigations and designing demanding security systems. A traffic accident may cause not only damages to the health and lives of persons involved in the traffic accident, but also damage to the general public utility equipment - road, traffic signs, crash barriers, fencing, buildings. A traffic accident may also cause fire and the release of dangerous substances. A separate but closely related chapter is the evaluation of accidents or collision situations when monitoring the operation of vehicles with priority driving. According to the survey, about a third of users (33\%), who have submitted video recording from dashcam as part of the claim process, rated this process as very simple and a quarter of users $(25 \%)$ as a quick process.

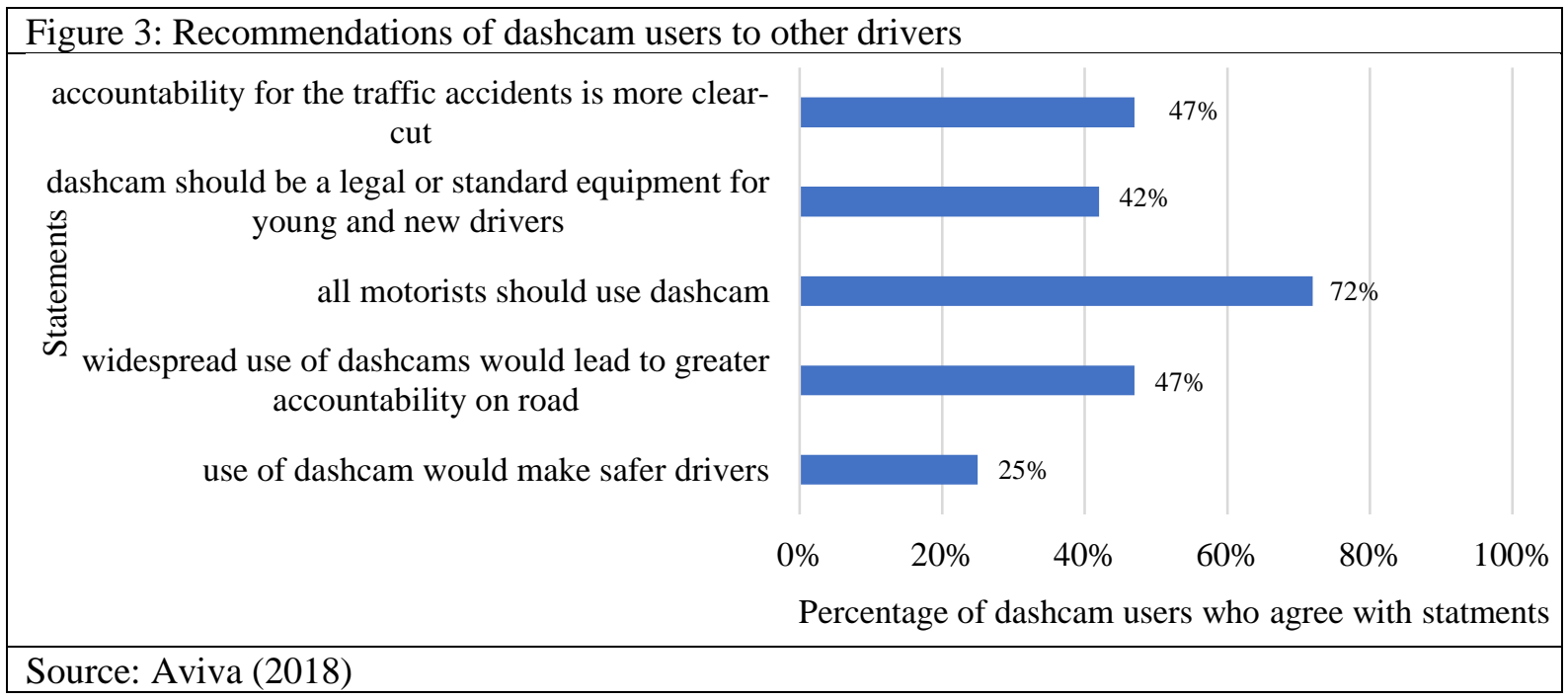

Figure 3 shows the percentage redistribution of dashcam users who give recommendations to other drivers. The respondents agree with more than one option. In general, the statements of dashcam users have a positive recommended character and these drivers agree that the dashcam should become standard equipment in a vehicle. Using a dashcam can bring benefits mainly to young and new drivers, such as a digital accident video-recording, objective information of the course of collision, greater accountability and so on. 


\section{Conclusion}

The dashcam is a road phenomenon and important preventive and safety element. Global benefits include the ability to see directly from the optical digital evidence what happened during the accident, which means that the inaccuracies of the accident reconstruction are eliminated. Given the growing trend in the use of dashcams (Dashboard Camera Market Size, 2020), I would like to focus in my future research work mainly on the requirements imposed on these devices. Some of these were mentioned in the previous part. The requirements are the parameters on the basis of which the end user selects the dashcam. Limits were found in relation to the mechanical resistance. It is very important for the dashcam to record and save video recordings in events of high acceleration/deceleration due to vehicle impact or due to direct impact force on the camera body. At present, there is no uniform methodology for testing this parameter and mechanical resistance mentioned in the relevant standard covers only a part of possible traffic accident scenarios. The benefits of research in this particular parameter can have a positive impact in determining the suitability of using dashcams, finding out the possibility of accurately interpreting the obtained data, the possibility of their use to increase the legal certainty of the population and minimizing the level of insurance fraud and other illegal actions.

\section{References}

Aviva. (2018, Jul 02). 11 million UK motorists keep a digital eye on the road. Retrieved from https://www.aviva.com/newsroom/news-releases/2018/07/dash-for-dash-cam/

Bouwmans, T., Porikli, F., Hoferlin, B., Vacavant, A. (2014). Background Modeling and Foreground Detection for Video Surveillance. In: CRC Press, 2014.

Boroš, M., Vel'as, A., Ďurovec, M. (2018). Research of principles used for video motion detection in video surveillance systems. In: WIT Transactions on the Built Environment, 174, pp. 297-302. DOI: 10.2495/SAFE170271.

Dashboard Camera Market Size, Industry Report, 2020-2027. (2020). Retrieved from https://www.grandviewresearch.com/industry-analysis/dashboard-camera-market

Gusner, P. (2014). 7 reasons to use a dashboard camera. Retrieved from http://www.insurance.com/auto-insurance/claims/7reasons-to-use-a-dash-cam.html

Han, I. (2016). Car speed estimation based on cross-ratio using video data of car-mounted camera (black box). In: Forensic Science International 269, pp. 89-96. DOI: 10.1016/j.forsciint.2016.11.014.

Ivor, J., Kolla, E. (2019). Záznam z autokamery ako dôkaz v trestnom konaní (Vehicle Camera Record as the Proof in Criminal Proceedings). In: Informačno-technické prostriedky v trestnom konaní - možnosti a perspektívy, 2019- April, pp. 92-108. ISBN 978-80-7502-363-6.

Jones, M. (2014). Dash Cams - What Are The Benefits? Retrieved from http://blog.extended-vehiclewarranty.com/blog/dash-cams

Kadu, S., Cheggoju, N., Satpute, V.R. (2018). Noise-resilient compressed domain video watermarking system for in-car camera security. In. Multimedia Systems, 2018-October, pp. 583-595. DOI: 10.1007/s00530-017-0584-3.

Kolla, E., Ondruš, J., Vertal', P. 2019. Reconstruction of traffic situations from digital video-recording using method of volumetric kinetic mapping. In: he Archives of Automotive Engineering - Archiwum Motoryzacji, pp 147-170. DOI: 10.14669/AM.VOL84.ART11.

Lavrinc, D. (2013). Why Almost Everyone in Russia Has a Dash Cam? Retrieved from http://www.wired.com/2013/02/russian-dash-cams

Ma, B., Reibman, A. R. (2017). DashCam video compression using historical data. In: Picture Coding Symposium, 2019December. DOI: 10.1109/PCS.2016.7906326.

Mehrish, A., Subramanyam, A.V., Kankanhalli, M. (2017). Multimedia signatures for vehicle forensics. In: IEEE International Conference on Multimedia and Expo, 2017-July, pp. 685-690. DOI: 10.1109/ICME.2017.8019488.

Pettie, E. (2013). A Brief History of Dash Cam Insanity. Retrieved from http://www.break.com/article/a-brief-history-of-thedash-cam-2425146

Russo, G., Baccaglini. E., Boulard, L., Brevi, D., Scopigno, R. (2015). Video processing for V2V communications: A case study with traffic lights and plate recognition. In: 1st IEEE International Forum on Research and Technologies for Society and Industry, 2015-Noveber, pp. 144-148. DOI: 10.1109/RTSI.2015.7325064.

Šoltés, V., Mariš, L., Lenko, F. (2019). Impact of selected traffic accidents in regions on citizens' safety. In: Transport Means - Proceedings of the International Conference, 2019-October, pp. 979-982.

Štitilis. D., Laurnaitis. M. (2016). Legal regulation of the use of dashboard cameras: Aspects of privacy protection. In: Computer Law \& Security Review, 2016-April. pp. 316-326.

Torney, Ch. (2014). Cheaper cover for drivers with dashcams. Retrieved from http://www.confused.com/carinsurance/articles/the-insurer-offering-discounts-to-drivers-with-dashcams

Wong, T.W., Tao, C.H., Cheng, Y.K., Wong, K.H., Tam, C.N. (2014). Application of cross-ratio in traffic accident reconstruction. In: Forensic Science International 235, pp. 19-23. DOI: 10.1016/j.forsciint.2013.11.012. 